\title{
Nonadiabatic Surface Reaction: Mechanism of Electron Emission in the $\mathrm{Cs}+\mathrm{O}_{2}$ System
}

\author{
A. Böttcher, R. Imbeck, A. Morgante, and G. Ertl \\ Fritz-Haber-Institut der Max-Planck-Gesellschaft, Faradayweg 4-6, D-1000 Berlin 33, West Germany
}

(Received 23 April 1990)

\begin{abstract}
Electron emission during oxidation of thin $\mathrm{Cs}$ films is confined to the stage $\mathrm{Cs}_{2} \mathrm{O}_{2} \rightarrow \mathrm{CsO}_{2}$ and is caused by Auger deexcitation accompanying the (formal) reaction $\mathrm{O}_{2}{ }^{2-}(s)+\mathrm{O}_{2} \rightarrow 2 \mathrm{O}_{2}{ }^{-}(s)$. This nonadiabatic process becomes possible because resonance ionization of the affinity level of the impinging $\mathrm{O}_{2}$ molecule upon crossing the Fermi level $E_{F}$ is efficiently suppressed due to the absence of occupied states near $E_{F}$ at the surface.
\end{abstract}

PACS numbers: $79.20 . \mathrm{Nc}$, 68.35.Fx

Nonadiabatic chemical reactions, i.e., processes for which the nuclear motion is not restricted to the electronic ground state of the system, are expected to occur less frequently with molecule- (metal-) surface interactions than with homogeneous gas phase reactions, because of the generally higher probability of electronic quenching in the former case. There exist, however, several examples which indicate that at least part of the reaction exothermicity is transferred into electronic excitation which subsequently is released, e.g., in the form of light emission ${ }^{1-3}$ or electron ejection. ${ }^{2-6}$ Although theoretical models for the mechanism of exoelectron emission have been proposed in the literature, ${ }^{3,6-9}$ the existing experimental evidence is still scarce and renders this effect somewhat exotic. This Letter reports on a study of exoelectron emission accompanying the interaction of $\mathrm{O}_{2}$ with $\mathrm{Cs}$ films which, due to combination with information from electron spectroscopies, enabled for the first time a clear identification of the underlying physical processes and interpretation in terms of previously proposed theoretical concepts.

The experiments were performed with an UHV system containing [apart from LEED-Auger and thermaldesorption spectroscopy (TDS) as standard tools] a hemispherical electron energy analyzer and facilities for ultraviolet photoelectron spectroscopy (UPS) and metastable deexcitation spectroscopy (MDS). ${ }^{10}$ For surfaces with low work functions, the latter technique is based on Auger deexcitation of impinging metastable noble gas atoms, whose excitation energy (e.g., $19.8 \mathrm{eV}$ for ${ }^{3} S$ $\mathrm{He}^{*}$ ) serves to emit electrons from the target. The kinetic-energy distribution of these electrons is measured and reflects the local density of occupied states of the outermost atomic layer of the solid. Thin Cs films were evaporated onto a $\mathrm{Ru}(0001)$ substrate, and the experiments described below were performed with films of about 2-3-monolayers thickness and at substrate temperatures around $220 \mathrm{~K}$.

Figure 1, curve $a$, shows the yield of electrons emitted as a function of the $\mathrm{O}_{2}$ exposure at a partial pressure of $4 \times 10^{-9}$ mbar. If one compares the integral electronic charge emitted with the total uptake of $\mathrm{O}_{2}$ molecules (as estimated from the amount of Cs atoms determined by
TDS, and assuming complete transformation into $\mathrm{CsO}_{2}$ ), an efficiency of the order of roughly $10^{-3}$ results. Interestingly, no electron emission can be detected upon interaction of $\mathrm{O}_{2}$ with a clean $\mathrm{Cs}$ surface; the yield rises only after about $1 \mathrm{~L}\left(1 \mathrm{~L}=10^{-6}\right.$ Torrs) exposure, i.e., after partial oxidation. This observation is in qualitative agreement with previous findings, e.g., with the $\mathrm{O}_{2}+\mathrm{Mg}$ system. $^{2,11}$ It was suggested that an increase of electron yield is generally caused by the decrease of the work function (following a third power law). ${ }^{3}$ Inspection of curve $b$ in Fig. 1 shows the concurrently recorded variation of the work function $\phi$, which indeed decreases with increasing $\mathrm{O}_{2}$ exposure to a minimum from where it rises again. However, the electron yield shows a marked increase definitely only beyond the work-function minimum, i.e., parallel to an increase of $\phi$. (As will become evident later, a work-function effect of the quoted type indeed plays a role at even higher $\mathrm{O}_{2}$ exposures when the electron yield drops again.) This result suggests that the emission of electrons is governed rather by the chemical nature, i.e., the valence electronic structure, of the system.

Interaction of $\mathrm{Cs}$ with $\mathrm{O}_{2}$ proceeds via the formation of suboxides with the $\mathrm{Cs}_{11} \mathrm{O}_{3}$ structural unit ${ }^{12}$ to the peroxide $\mathrm{Cs}_{2} \mathrm{O}_{2}$ and finally to the superoxide $\mathrm{CsO}_{2}$. ${ }^{13,14}$

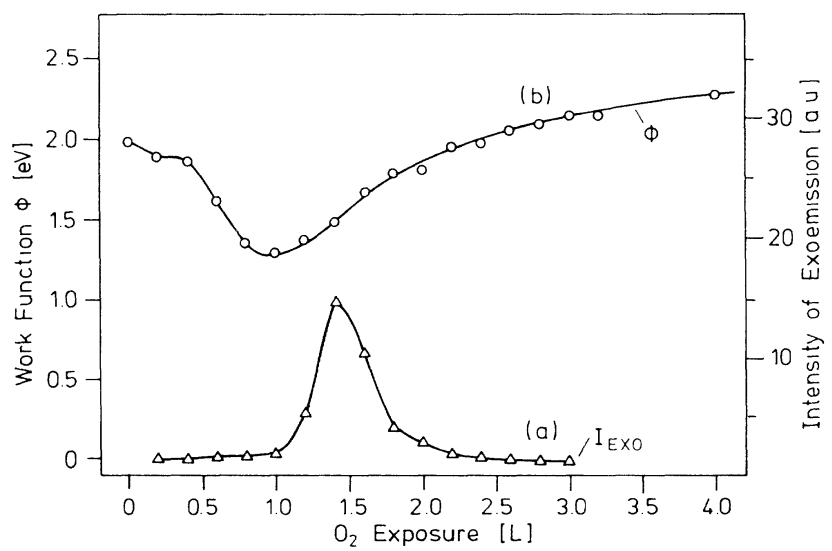

FIG. 1. Yield of exoelectrons (curve $a$ ) and work function $\phi$ (curve $b$ ) of a thin Cs film as a function of $\mathrm{O}_{2}$ exposure. 
The work-function minimum is reached near completion of the $\mathrm{Cs}_{2} \mathrm{O}_{2}$ phase at the surface, while the subsequent $\phi$ increase parallels further transformation into $\mathrm{CsO}_{2} .{ }^{14}$ The data of Fig. 1 suggest that just this stage, i.e., the process

$$
\mathrm{Cs}_{2} \mathrm{O}_{2} \stackrel{\mathrm{O}_{2}}{\rightarrow} \mathrm{CsO}_{2}
$$

gives rise to noticeable exoelectron emission.

In order to corroborate this idea, the variation of the electronic structure of the outermost atomic layer (such as that also "seen" by the incoming $\mathrm{O}_{2}$ molecules) was monitored by MDS while the yield of exoelectrons was recorded in parallel. The resulting data are reproduced in Fig. 2. The MDS data [Fig. 2(a)] are in complete
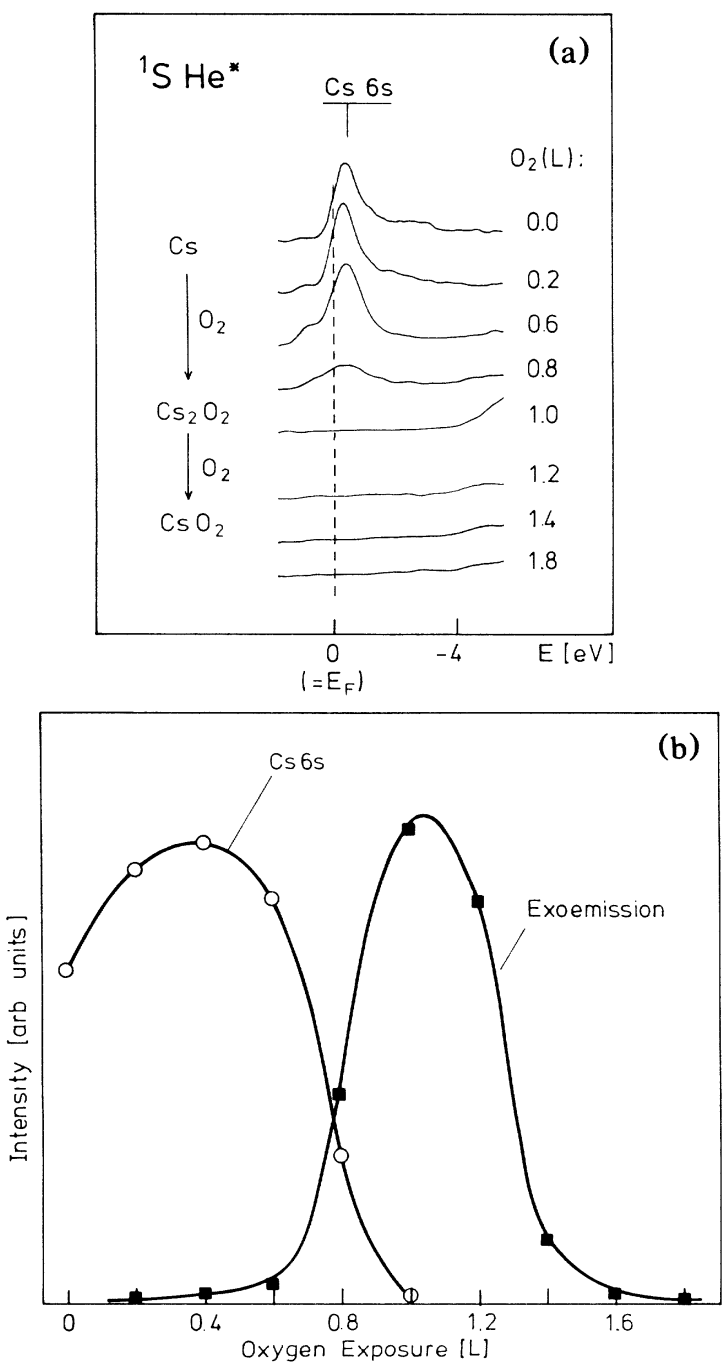

FIG. 2. (a) Metastable deexcitation spectroscopy (MDS) data from the valence-band region of a thin Cs film under the influence of progressing $\mathrm{O}_{2}$ exposure. [Note that the smallintensity contributions above $E_{F}$ are due to incomplete singlet to triplet conversion of the incoming $\mathrm{He}^{*}$ (Ref. 14).] (b) Variation of the intensities of Cs $6 s$ emission as probed by MDS, and of exoelectron emission as a function of $\mathrm{O}_{2}$ exposure. agreement with those from a previous more detailed study ${ }^{14}$ and are interpreted as follows: The clean Cs surface exhibits an intense band from $6 s$ states right below the Fermi level whose intensity even increases with small $\mathrm{O}_{2}$ doses. This effect is associated with the formation of $\mathrm{Cs}_{11} \mathrm{O}_{3}$ suboxide units for which the $\mathrm{O}^{2-}$ ions penetrate below the surface and cause a quantum-size effect for the conduction electrons of the outermost, still metallic, Cs atoms. ${ }^{15}$ Only beyond $\sim 0.4-\mathrm{L} \mathrm{O}_{2}$ exposure does the Cs $6 s$ emission start to decrease in intensity due to the onset of the formation of $\mathrm{Cs}_{2} \mathrm{O}_{2}$ at the surface; this compound consists of $\mathrm{Cs}^{+}$ions (with empty $6 s$ levels) and peroxide ions $\mathrm{O}_{2}{ }^{2-}$. This process is then followed by transformation into $\mathrm{CsO}_{2}$, exhibiting $\mathrm{Cs}^{+}$and hyperoxide ions $\mathrm{O}_{2}{ }^{-}$. As can be seen from Fig. 2(b), the decrease of the intensity of the Cs $6 s$ emission as probed by MDS directly parallels the increase of exoelectron emission which reaches its maximum at 1.0 -L exposure, just when occupied Cs $6 s$ levels close to $E_{F}$ have completely disappeared. (The data for Figs. 1 and 2 were taken with films of different thickness. Since bulk diffusion plays a role in the oxidation process, this affects the exposures at which the maximum of exoelectron emission is reached.)

The mechanism governing electron emission in this surface reaction is interpreted in terms of the model proposed by Kasemo et al. ${ }^{7}$ as depicted in Fig. 3. When $\mathrm{O}_{2}$ approaches the surface, its affinity level, which for the free molecules is located at $E_{A}=0.4 \mathrm{eV}$ below the vacuum level, will experience a downward shift, at first because of image force effects at larger distances and then by chemical interaction (i.e., bond formation) until it reaches an energetic position $\varepsilon_{A}$. A normal (adiabatic) transformation will be characterized by the filling of this level as soon as it crosses the Fermi level of the substrate. If, for any reason, the $\mathrm{O}_{2}$ molecule reaches the surface without the level at $\varepsilon_{A}$ being occupied, this hole state may be deexcited in an Auger process as indicated. The excited electron may escape the solid with a maximum kinetic energy (as referred to the vacuum level) $\varepsilon_{\text {kin,max }}^{\prime}=E_{F}-\varepsilon_{A}-\phi$, or if $\varepsilon_{A}$ and $\varepsilon_{\text {kin }}$ are scaled with respect to the Fermi level, $E_{F}=0: \varepsilon_{\max }\left(E_{F}\right)=-\varepsilon_{A}$.

We identify $\varepsilon_{A}$ with the energy of the highest occupied $\mathrm{O} 2 p$-derived level of the $\mathrm{Cs}_{2} \mathrm{O}_{2} / \mathrm{CsO}_{2}$ system which is probed by UPS at a binding energy of about $-2.5 \mathrm{eV}$ (see also Refs. 13 and 14). [This level does not show up

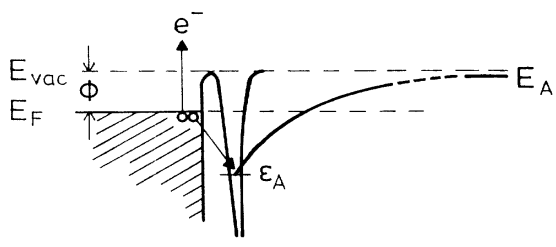

FIG. 3. Schematic diagram illustrating the mechanism of electron emission following the theoretical model proposed by Kasemo et al. (Ref. 7) 
in the MDS data of Fig. 2(a), indicating that it is "buried" below the topmost layer of charge density.] Hence we expect $\varepsilon_{\max }\left(E_{F}\right) \approx 2.5 \mathrm{eV}$. Kinetic-energy distributions of exoelectrons recorded at various stages beyond the intensity maximum (cf. Figs. 1 and 2) are reproduced in Fig. 4 and are indeed in agreement with this prediction. Moreover, these distributions clearly exhibit a common leading edge at the high-kinetic-energy side, indicating identical $\varepsilon_{A}$, which also means the same chemical nature of the process causing electron emission. This confirms the suggestion that exoelectron emission is coupled to the reaction

$$
\mathrm{Cs}_{2} \mathrm{O}_{2} \stackrel{\mathrm{O}_{2}}{\rightarrow} \mathrm{CsO}_{2},
$$

or formally $\mathrm{O}_{2}{ }^{2-}(s)+\mathrm{O}_{2} \rightarrow 2 \mathrm{O}_{2}{ }^{-}(s)$.

The minimum kinetic energy with respect to the vacuum level has, of course, to be zero and hence with respect to the Fermi level $\varepsilon_{\max }\left(E_{F}\right)=\phi$. The open circles in Fig. 4 mark the magnitude of the work function, as derived from the total width of the UPS spectrum, belonging to the respective exoelectron energy distribution curve. These points closely coincide with the onsets of emission, which are broadened because of the limited resolution of the electron energy analyzer. These data indicate that with increasing $\phi$ also an increasing fraction of electrons excited to levels above $E_{F}$ will be prevented from escaping into the vacuum. Hence the decrease in total emission will be further reduced by the decrease in reaction probability upon approaching completion of the oxide layer formation.

A crucial prerequisite for the proposed mechanism is that the empty affinity level of the incoming $\mathrm{O}_{2}$ molecule "survives" the crossing of the Fermi level and captures an electron only if it is close enough to the surface to have reached its equilibrium value $\varepsilon_{A}$. The lifetime $\tau_{\mathrm{el}}$ of this empty state after crossing the Fermi level is inversely proportional to its width $\Delta$. The latter in turn is governed by $V_{a k}=\left\langle a\left|H_{e}\right| k\right\rangle$, which means essentially by the overlap between the wave function $\langle a|$ of the empty affinity level of the incoming particle and that of the occupied states $|k\rangle$ near $E_{F}$ of the solid. ${ }^{9}$ As long as an impinging $\mathrm{O}_{2}$ molecule can "see" occupied Cs $6 s$-derived states, rapid resonance ionization will take place upon the crossing of its affinity level with $E_{F}$ and the system will follow the adiabatic potential curve without electronic excitation. Only at those sites at which transformation into $\mathrm{Cs}^{+}\left(=\mathrm{Cs}_{2} \mathrm{O}_{2}\right)$ has already been completed, and hence no occupied states close to $E_{F}$ are available, the lifetime will become long enough to permit appreciable electronic excitation to be followed by Auger decay and eventually exoelectron emission. It is likely that the low total probability for this process is mainly caused by the fact that a large fraction of the excited electrons will not have high enough kinetic energy to overcome the work-function barrier, as is suggested if the data of Fig. 4 are tentatively extrapolated. Other factors limiting the efficiency of electron emission have to be explored in fu-

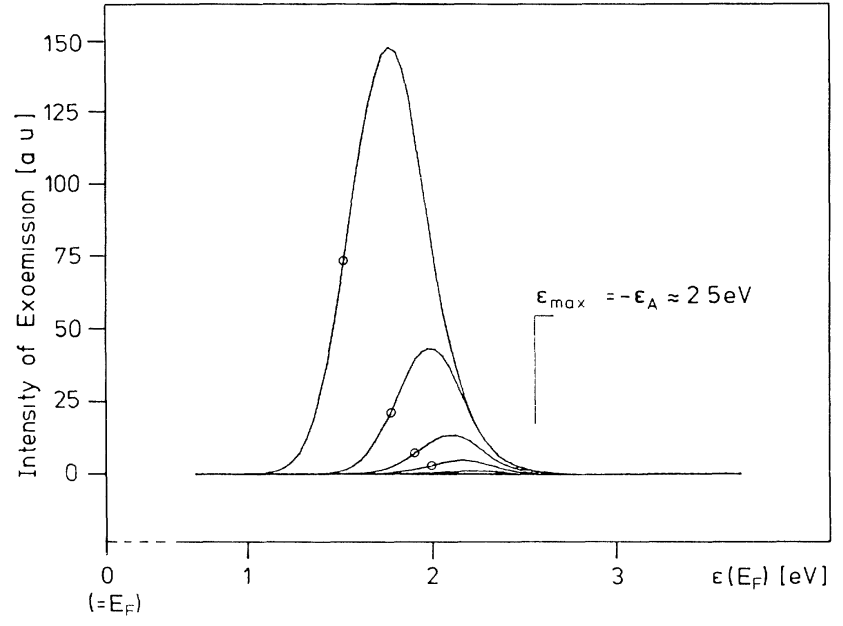

FIG. 4. Kinetic-energy distributions of the exoelectrons emitted at various stages of oxidation beyond the workfunction minimum. Circles mark the corresponding values of $\phi$. The energy scale is referred to the Fermi level $E_{F}$.

ture work.

In summary, a consistent picture for the mechanism of electron emission during nonadiabatic oxidation of thin Cs films has been presented. It is based on previously proposed theoretical concepts and explains all the experimental observations, such as the dependence of the electron yield on the electronic structure of the surface (as defined by its chemical nature) as well as the kineticenergy distribution of the emitted electrons.

'B. Kasemo, Phys. Rev. Lett. 32, 1114 (1974).

${ }^{2}$ G. C. Allen, P. M. Tucker, B. E. Hayden, and D. F. Klemperer, Surf. Sci. 102, 207 (1981).

${ }^{3}$ R. H. Prince, R. M. Lambert, and J. S. Foord, Surf. Sci. 107, 605 (1981).

${ }^{4}$ T. F. Gesell, E. T. Arakawa, and T. A. Callcott, Surf. Sci. 20, 174 (1970)

${ }^{5}$ M. P. Cox, J. S. Foord, R. M. Lambert, and R. H. Prince, Surf. Sci. 129, 399 (1988).

${ }^{6}$ R. H. Prince and R. Persaud, Surf. Sci. 207, 207 (1988).

${ }^{7}$ B. Kasemo, E. Törnquist, J. K. Nørskov, and B. I. Lundqvist, Surf. Sci. 89, 554 (1979).

${ }^{8}$ J. K. Nørskov, D. M. Newns, and B. I. Lundqvist, Surf. Sci. 80, 179 (1979).

${ }^{9}$ J. K. Nørskov, J. Vac. Sci. Technol. 18, 420 (1981).

${ }^{10}$ H. Conrad, G. Ertl, J. Küppers, W. Sesselmann, and H. Haberland, Surf. Sci. 121, 161 (1982).

${ }^{1}$ T. F. Gesell and E. T. Arakawa, Surf. Sci. 33, 419 (1972).

${ }^{12}$ A. Simon, J. Solid State Chem. 27, 87 (1979).

${ }^{13}$ C. Y. Su, I. Lindau, P. W. Chye, S.-J. Oh, and W. E. Spicer, J. Electron. Spectrosc. 31, 221 (1983).

${ }^{14}$ B. Woratschek, W. Sesselmann, J. Küppers, G. Ertl, and H. Haberland, J. Chem. Phys. 86, 2411 (1987).

${ }^{15}$ B. Woratschek, G. Ertl, J. Küppers, W. Sesselmann, and H. Haberland, Phys. Rev. Lett. 57, 1484 (1986). 